\title{
LINE-GEOMETRIC REPRESENTATIONS FOR FUNCTIONS OF A
}

\author{
COMPLEX VARIABLE.*
}

BY

\section{E. J. WILCZYNSKI}

\section{INTRODUCTION}

The usefulness of the usual method of analytic geometry, for visualizing a functional relation between two real variables, is too well known to call for further comment. A second method, not quite so familiar, has also been used extensively, especially in projective geometry. It consists in constructing scales on two straight lines, one for the independent and one for the dependent variable, joining corresponding points of the two scales by straight lines, and studying the one-parameter family of straight lines obtained in this way.

This second method can be utilized also for the purpose of visualizing a functional relation between two complex variables. If $z=x+i y$, and $w=u+i v$ are two complex variables, and if $w$ is a function of $z$, we may proceed as follows. Choose any two planes of ordinary space as the planes of the two complex variables, and join by straight lines the points which corspond to each other in the functional relation. The result will be a twoparameter family of lines, a congruence, and this congruence may be regarded as a geometric image of the functional relation. It should be mentioned immediately, however, that the properties of this congruence depend, not merely on the properties of the function $w=F(z \cdot)$, but also upon the relative position of the two planes. We shall, in this paper, consider only the case which suggests itself most naturally, namely the case of two parallel planes. Only in this case will all of the points which represent finite values of $z$ be geometrically distinct from those which represent finite values of $w$.

Weierstrass, in a very brief and incidental manner, mentions the possibility of such a line-geometric image of a functional relation. $\dagger$ But he did not investigate the properties of the resulting congruences; in fact he disposes of the whole matter in a very few words. There exists, however, an extensive

* Presented to the Society, December 28, 1918, and April 4, 1919.

† K. Weierstrass, Mathematische Werke. Vorlesungen über die Theorie der Abel'schen Transcendenten, Chapter 16, p. 323.

Trans. Am. Math. Soc. 19 
paper on this subject by M. J. Van Uven.* The work of Van Uven is devoted entirely to the discussion of special cases, such as $w=z^{n}, w=z^{-n}$, etc.; these are investigated in most thorough fashion, but the general questions are not attacked. A very interesting paper by Emch $†$ makes use of a method which is applicable only to the case of a linear function. But, in spite of this limitation, Emch's paper is important, because in his paper the planes of the two complex variables are not parallel. Thus we are in a position to see, by comparing Emch's results with our own, that the mutual position of the two planes is an important feature of the theory. In fact we shall see that, for parallel planes, related to each other as described in Article 1, the congruence always has imaginary cones as focal surfaces, while Emch obtains real nondevelopable focal surfaces for the congruence which corresponds to the case which he has studied.

In Article 2 we shall present a second method of representing $w=F(z)$ by means of a congruence. This method consists in interpreting both variables as points of the same Riemann sphere, and then joining corresponding points by straight lines. The focal surfaces of the congruences obtained by this method are always real and possess some very striking properties. The possibility of such a representation seems to have occurred to Study, $\ddagger$ but he did not develop the idea in detail. The method of Coolidge, $\S$ which makes use of congruences in non-euclidean (elliptic) space, is different from any of these and far less elementary.

Let us write

\section{The method of parallel planes}

$$
x+i y=z, \quad x-i y=z_{0}, \quad u+i v=w, \quad u-i v=w_{0},
$$

and let us assume that $w$ is a function of $z$, say

so that

$$
w=F(z),
$$

$$
w_{0}=F_{0}\left(z_{0}\right) \text {, }
$$

where $F_{0}$ denotes the function conjugate to $F$.

We interpret $z=x+i y$ in the usual way as a point $P_{z}$ of the $\xi \eta$ plane, so

* M. J. Van Uven, Algebraische Strahlencongruenzen und verwandte komplexe Ebenen als Schmitte derselben. Verhandelingen der Konniklijke Akademie van We tens chappen te Amsterd a m (Eerste Sectie), vol. 10 (1910), pp. 1-527.

$\dagger$ A. Emch, On the rectilinear congruence realizing a circular transformation of one plane into another, An a ls of M a thematics, ser. 2, vol. 13 (1911-12), p. 155.

Study, Über Nicht-Euklidische und Linien-Geometrie, $\mathrm{J}$ a h r e s b e r i c h t d e r Deutschen Mathematiker Vereinigung, vol. 11 (1902), p. 329.

$\S \mathrm{J}$. L. Coolidge, Les congruences isotropes qui servent d représenter les fonctions d'une variable complexe, A t t i di Torino, vol. 38 . 
that the cartesian coördinates of $P_{z}$ will be

$$
\xi_{1}=x, \quad \eta_{1}=y, \quad \zeta_{1}=0 .
$$

We place the plane of the complex variable $w$ parallel to that of $z$, in such a way that the origin of the $w$-plane shall be on the $\zeta$-axis at the point $(0,0,1)$, the $u$ - and $v$-axes being parallel to the $x$ - and $y$-axes respectively. Then the cartesian coördinates of $P_{w}$ will be

$$
\xi_{2}=u, \quad \eta_{2}=v, \quad \zeta_{2}=1 .
$$

Let the homogeneous cartesian coördinates of $P_{z}$ and $P_{w}$ be $\left(\lambda_{1}, \cdots \lambda_{4}\right)$. and $\left(\mu_{1}, \cdots \mu_{4}\right)$ respectively. If we express $x, y, u$, and $v$ in terms of $z, z_{0}, w, w_{0}$, by means of (1), we may write

$$
\begin{array}{llll}
\lambda_{1}=\frac{1}{2}\left(z+z_{0}\right), & \lambda_{2}=\frac{1}{2 i}\left(z-z_{0}\right), & \lambda_{3}=0, & \lambda_{4}=1, \\
\mu_{1}=\frac{1}{2}\left(w+w_{0}\right), & \mu_{2}=\frac{1}{2 i}\left(w-w_{0}\right), & \mu_{3}=1, & \mu_{4}=1,
\end{array}
$$

where, of course, only the ratios $\lambda_{i}: \lambda_{k}$ and $\mu_{i}: \mu_{k}$ are of significance.

In order to study the congruence, which is obtained when we join corresponding points $P_{z}$ and $P_{w}$, we make use of a system of linear homogeneous differential equations satisfied by each of the four pairs of functions $\left(\lambda_{k}, \mu_{k}\right)$. This system of partial differential equations will be composed of two equations of the first order, and of two equations of the second order. The second order equations are obtained very easily. Obviously the equations

$$
\frac{\partial^{2} \lambda}{\partial z^{2}}=0, \quad \frac{\partial^{2} \lambda}{\partial z_{0}^{2}}=0, \quad \frac{\partial^{2} \mu}{\partial w^{2}}=0, \quad \frac{\partial^{2} \mu}{\partial w_{0}^{2}}=0
$$

are satisfied by each of the eight functions $\lambda_{k}, \mu_{k}$. The latter two assume the form

$$
\frac{\partial^{2} \mu}{\partial z^{2}}-\frac{w^{\prime \prime}}{w^{\prime}} \frac{\partial \mu}{\partial z}=0, \quad \frac{\partial^{2} \mu}{\partial z_{0}}-\frac{w_{0}^{\prime \prime}}{w_{0}^{\prime}} \frac{\partial \mu}{\partial z_{0}}=0,
$$

if we introduce $z$ and $z_{0}$ as independent variables, and if we write

$$
w^{\prime}=\frac{d w}{d z}, \quad w^{\prime \prime}=\frac{d^{2} w}{d z^{2}}, \quad w_{0}=\frac{d w_{0}}{d z_{0}}, \quad w^{\prime \prime}=\frac{d^{2} w_{0}}{d z_{0}^{2}} .
$$

In this connection the following remark is essential. If we maintain our original agreement that $z$ and $z_{0}$ are conjugate complex variables, that is, that $x$ and $y$ are real, we can not treat $z$ and $z_{0}$ as independent complex variables. Consequently we generalize our problem, at this stage, by thinking of $x$ and $y$ as independent complex variables. In most of the applications, however, we return to our original hypothesis, that $x$ and $y$ are real.

Direct substitution of the four pairs of functions $\left(\lambda_{k}, \mu_{k}\right)$ enables us to 
verify that each of these pairs also satisfies the two first order equations

Let us put

$$
w^{\prime} \frac{\partial \lambda}{\partial z}-\frac{\partial \mu}{\partial z}=0, \quad w_{0}^{\prime} \frac{\partial \lambda}{\partial z_{0}}-\frac{\partial \mu}{\partial z_{0}}=0 .
$$

$$
\bar{\lambda}=w^{\prime} \lambda-\mu, \quad \bar{\mu}=w_{0}^{\prime} \lambda-\mu,
$$

whence

$$
\left(w^{\prime}-w_{0}^{\prime}\right) \lambda=\bar{\lambda}-\bar{\mu}, \quad\left(w^{\prime}-w_{0}^{\prime}\right) \mu=w_{0}^{\prime} \bar{\lambda}-w^{\prime} \bar{\mu} .
$$

Then we may write, in place of (7),

$$
\frac{\partial \bar{\lambda}}{\partial z}=\frac{w^{\prime \prime}}{w^{\prime}-w_{0}^{\prime}}(\bar{\lambda}-\bar{\mu}), \quad \frac{\partial \bar{\mu}}{\partial z_{0}}=\frac{w_{0}^{\prime \prime}}{w^{\prime}-w_{0}^{\prime}}(\bar{\lambda}-\bar{\mu}),
$$

if we assume $w^{\prime}-w_{0}^{\prime} \neq 0$. The case thus excluded has little interest, since it corresponds to the case when $w^{\prime}$ is a real constant, so that $w=k z+b$ $(k$ real $)$. In this case the congruence degenerates into a bundle of lines.

We proceed to interpret equations (10). Under the assumption

$$
w^{\prime}-w_{0}^{\prime} \neq 0 \text {, }
$$

equations (8) give rise to two distinct points, $P_{\bar{\lambda}}$ and $P_{\bar{\mu}}$, on the line $P_{z} P_{w}$. Let $P_{z}$ move along one of the null lines $z_{0}=$ const. of the $x y$-plane. Then the line $P_{z} P_{w}$ generates an imaginary ruled surface of the congruence. The point $P_{\bar{\lambda}}$ describes a curve on this ruled surface whose tangent is obtained by joining $P_{\bar{\lambda}}$ to the point whose coördinates are $\partial \bar{\lambda}_{k} / \partial z$. According to (10) this tangent coincides with the line $P_{\bar{\lambda}} P_{\bar{\mu}}$, that is, with the line $P_{z} P_{w}$ itself. In other words, this ruled surface is a developable, and the locus of $P_{\bar{\lambda}}$ is its cuspidal edge. Moreover, since this developable contains a straight line (a null-line of the $\xi \eta$-plane), the cuspidal edge of the developable must be a plane curve, a fact which we shall also verify later analytically.

Thus, the imaginary ruled surfaces of the congruence, which correspond to the two families of null lines of the $\xi \eta$-plane, constitute the developables of the congruence. Each of these developables touches one of the sheets of the focal surface along a plane curve.

We can simplify the form of equations (10) by putting

$$
f=\frac{\bar{\lambda}}{w^{\prime}-w_{0}^{\prime}}, \quad g=\frac{\bar{\mu}}{w^{\prime}-w_{0}^{\prime}} .
$$

They then assume the form

$$
\frac{\partial f}{\partial z}=m g, \quad \frac{\partial g}{\partial z_{0}}=n f
$$

where

$$
m=\frac{-w^{\prime \prime}}{w^{\prime}-w_{0}^{\prime}}, \quad n=\frac{+w_{0}^{\prime \prime}}{w^{\prime}-w_{0}^{\prime}} .
$$

Since we are using homogeneous coördinates, $f$ and $g$ will represent the same 
points as $\bar{\lambda}$ and $\bar{\mu}$ respectively, namely the two foci of a line of the congruence, that is, the two points in which $P_{z} P_{w}$ touches the two sheets of the focal surface.

By means of (9) and (11), we find

$$
\lambda=f-g, \quad \mu=w_{0}^{\prime} f-w^{\prime} g .
$$

Equations (12) are the two first order differential equations, in the canonical form which is most convenient for the application of the general theory of congruences. The two second order equations may be obtained from (5) and (6) by introducing the variables $f$ and $g$ by means of (14). Two of the equations obtained in this way are

$$
\frac{\partial^{2} f}{\partial z_{0}^{2}}=\frac{\partial n}{\partial z_{0}} f+n \frac{\partial f}{\partial z_{0}}, \quad \frac{\partial^{2} g}{\partial z^{2}}=\frac{\partial m}{\partial z} g+m \frac{\partial g}{\partial z}
$$

the other two are consequences of (12) and (15).

Thus we have found that our four pairs of functions $\left(f_{k}, g_{k}\right)$ are solutions of a system of differential equations of the form*

where

$$
\begin{gathered}
\frac{\partial f}{\partial z}=m g, \quad \frac{\partial g}{\partial z_{0}}=n f, \\
\frac{\partial^{2} f}{\partial z_{0}^{2}}=a f+b g+c \frac{\partial f}{\partial z_{0}}+d \frac{\partial g}{\partial z}, \\
\frac{\partial^{2} g}{\partial z^{2}}=a^{\prime} f+b^{\prime} g+c^{\prime} \frac{\partial f}{\partial z_{0}}+d^{\prime} \frac{\partial g}{\partial z},
\end{gathered}
$$

$$
\begin{aligned}
& m=\frac{-w^{\prime \prime}}{w^{\prime}-w_{0}^{\prime}}, \quad n=\frac{+w_{0}^{\prime \prime}}{w^{\prime}-w_{0}}, \\
& a=\frac{\partial n}{\partial z_{0}}, \quad b=0, \quad c=n, \quad d=0, \\
& a^{\prime}=0, \quad b^{\prime}=\frac{\partial m}{\partial z}, \quad c^{\prime}=0, \quad d^{\prime}=m .
\end{aligned}
$$

In general, the locus of $P_{f}$, one of the sheets of the focal locus is a surface. It degenerates into a curve, if and only if $m$ is equal to zero. This is an obvious consequence of (16), since $f$ will then be a function of $z_{0}$ alone. But $m$ can vanish identically, only if $w$ is an integral linear function of $z$, and in that case $n$ also will vanish, and therefore all of the coefficients of (16) will be equal to zero. To investigate the nature of the focal locus in this case, we make use of equations (4) and (8), after making the substitutions

$$
w=\alpha z+\beta, \quad w_{0}=\alpha_{0} z_{0}+\beta_{0},
$$

* This is the form of the system upon which I have based the general projective theory of congruences. See Sur la théorie générale des congruences, Mémoires publies par la Classe des Sciences de l'Academie Royale de Belgique, 2 série, vol. 3 (1911). Hereafter quoted as Brussels Paper. 
where $\alpha$ and $\beta, \alpha_{0}$ and $\beta_{0}$, are constants, and where we shall assume $\alpha \neq \alpha_{0}$ so as to avoid the exceptional case $w^{\prime}=w_{0}^{\prime}$ whose significance has been pointed out already. A simple calculation shows that the focal locus consists of two straight lines, one through each of the circular points of the $\xi \eta$-plane.

In all other cases, $m$ and $n$ are different from zero, and the focal locus is composed of two surfaces. Equations (17) show that $c^{\prime}$ and $d$ are equal to zero. Consequently the focal surface of the congruence is composed of two developables.* Evidently the case $m=n=0$ may be included in this statement, if we agree to regard a straight line as a degenerate case of a developable. Since we have, according to (15),

$$
\frac{\partial^{2} f}{\partial z_{0}}=\frac{\partial n}{\partial z_{0}} f+n \frac{\partial f}{\partial z_{0}},
$$

the curves on the sheet $S_{f}$ of the focal surface, which correspond to the null lines $z=$ const. of the $\xi \eta$-plane, are straight lines. We have seen already that the curves on $S_{f}$ which correspond to the null-lines $z_{0}=$ const. are plane curves.

The first part of this theorem becomes quite obvious, if we remember that the two families of developables of the congruence must determine a conjugate system of curves on each sheet of the focal surface. In our case the focal surfaces are developable, and on a developable one of the component families of a conjugate system always consists of its generators.

If we put

$$
h=\frac{\partial f}{\partial z_{0}}-n f,
$$

and make use of (16) and (17), we find

$$
\frac{\partial h}{\partial z}=0, \quad \frac{\partial h}{\partial z_{0}}=0 .
$$

But clearly the point $P_{h}$, whose coördinates $\left(h_{1}, \cdots, h_{4}\right)$ are given by (19), is on that generator of the developable $S_{f}$ which corresponds to a given constant value of $z$. According to (20), this point $P_{h}$ is the same for all such generators; consequently the focal surface $S_{f}$ is a cone. We may write

$$
h=\frac{\partial f}{\partial z_{0}}-\frac{\partial g}{\partial z_{0}}=\frac{\partial \lambda}{\partial z_{0}} .
$$

If we substitute the values (4) for $\lambda_{1}, \lambda_{2}, \lambda_{3}, \lambda_{4}$, we find

$$
h_{1}: h_{2}: h_{3}: h_{4}=i:-1: 0: 0 .
$$

Therefore, the vertex of the focal cone $S_{f}$ is one of the circular points of the $\xi \eta$-plane.

The curves $z=$ const. of $S_{f}$ are the generators of the cone. The curves $z_{0}=$ const. of $S_{f}$ are plane curves, a fact which we have mentioned before,

* Brussels Paper, p. 28. 
but which may also be proved as follows. We have

$$
\begin{gathered}
\frac{\partial f}{\partial z}=m g, \quad \frac{\partial^{2} f}{\partial z^{2}}=\frac{\partial m}{\partial z} g+m \frac{\partial g}{\partial z} \\
\frac{\partial^{3} f}{\partial z^{3}}=\left(\frac{\partial^{2} m}{\partial z^{2}}+m \frac{\partial m}{\partial z}\right) g+\left(2 \frac{\partial m}{\partial z}+m^{2}\right) \frac{\partial g}{\partial z} .
\end{gathered}
$$

From these three equations, we may eliminate $g$ and $\partial g / \partial z$. Thus we obtain the linear homogeneous differential equation of the third order for $f$;

$$
\frac{\partial^{3} f}{\partial z^{3}}-\left(m+2 \frac{\partial \log m}{\partial z}\right) \frac{\partial^{2} f}{\partial z^{2}}-\left[\frac{\partial^{2} \log m}{\partial z^{2}}-\left(\frac{\partial \log m}{\partial z}\right)^{2}\right] \frac{\partial f}{\partial z}=0
$$

Since $f_{1}, f_{2}, f_{3}, f_{4}$ are solutions of this third order equation, the curves $z_{0}=$ const. on $S_{f}$ are indeed plane curves. Moreover equation (22) may serve for the purpose of discussing the projective properties of these curves, and therefore of the focal cone. But, unless we specialize the function $w=F(z)$, we need not expect to find any special properties of the focal cone beyond those already noted. In fact we shall show that this focal cone may be regarded as the most general analytic cone which has its vertex at $(i,-1,0,0)$.

To prove this we observe in the first place that the cone $S_{f}$ is the envelope of the one-parameter family of planes

$$
x_{1}+i x_{2}+[z-F(z)] x_{3}-z x_{4}=0,
$$

all of which, of course, pass through the circular point $I(1, i, 0,0)$, which is the vertex of the cone. Now the most general one-parameter family of planes through $I$ may be represented by an equation of the form

$$
x_{1}+i x_{2}+\varphi(t) x_{3}+\psi(t) x_{4}=0 .
$$

If $\psi(t)$ does not reduce to a constant, we may put $\psi(t)=-z$, and then determine $F(z)$ by means of the equation

giving

$$
z-F(z)=\varphi(t),
$$

$$
F(z)=-\varphi(t)-\psi(t) .
$$

Thus, unless $\psi(t)=$ const., any family of form (24) is included in (23). If $\psi(t)$ is a constant, $\varphi(t)$ can not also be a constant if (24) is to represent a one-parameter family of planes. In the case $\psi(t)=$ const., (24) therefore represents a pencil of planes whose axis is a straight line in the $x y$-plane passing through $I$. This case only presents itself when $z$ regarded as a function of $w$ is a constant.

When the focal cone $S_{f}$ is given, the second focal cone $S_{f}$ is uniquely deter- 
mined. It is the envelope of the one-parameter family of planes

$$
x_{1}-i x_{2}+\left[z_{0}-F_{0}\left(z_{0}\right)\right] x_{3}-z_{0} x_{4}=0
$$

which are conjugate to (23).

Of course, the equations (23) and (25), regarded as simultaneous, are the equations of the two-parameter family of lines which constitute our congruence. In this form they are especially adapted to answer certain questions which arise naturally, especially when $F(z)$ is algebraic, as to the number of lines of the congruence which pass through a given point, or lie in a given plane, etc.

We may summarize our principal results as follows.

1. If $w=F(z)=$ const., the congruence reduces to a bundle of lines whose vertex is the point $w=$ const. of the $w$-plane. Similarly if $z=F^{-1}(w)=$ const.

2. If $w=k z+\beta$, where $k$ and $\beta$ are constants and where $k$ is real, the congruence still reduces to a bundle of lines, but its vertex does not lie in the plane of either complex variable.

3. If $w=\alpha z+\beta$, where $\alpha$ and $\beta$ are constants and where $\alpha$ is not real, the focal locus of the congruence is composed of two straight lines, one through each of the two circular points which the planes of $z$ and $w$ have in common.

4. In all other cases, the focal locus is composed of two cones whose vertices are the two circular points which the planes of $z$ and $w$ have in common. The developables of the congruence correspond to the null-lines of the two planes. Each of these developables is the locus of the tangents of a plane curve on one of the focal cones, and touches the other focal cone along a generator. The planes of the plane curves, thus determined on each of the focal cones, are the tangent planes of the other focal cone.

Interesting as these results are, they do not help us to visualize the properties of the function $w=F(z)$, except in the simple cases 1 and 2 , in which no such aid is needed. This is due to the fact that, in the general case, the developables and focal surfaces of the congruence are imaginary. In this respect the method of representation by means of the Riemann sphere (see Art. 2) is far superior. Nevertheless it is easy to utilize the method of parallel planes for furnishing real images of the functional relation. Thus every real curve of the z-plane determines a real ruled surface of the congruence which intersects the $w$-plane in a corresponding curve. These ruled surfaces, especially those which correspond to the lines $x=$ const. and $y=$ const., help us to understand the conformal correspondence between the two planes.* Moreover, certain loci connected with these ruled surfaces give rise to independent methods of defining the congruence.

We shall not, at present, discuss any questions of this general nature. We shall, however, indicate a method which enables us to replace the imaginary

* Van Uven's paper is largely devoted to the study of such ruled surfaces in the various special cases which he discusses. 
focal surfaces by certain real surfaces very closely connected with them. The foci, $P_{f}$ and $P_{g}$, of a line of the congruence are conjugate complex points of the line. Consequently the harmonic conjugate of $P_{z}$ with respect to $P_{f}$ and $P_{\sigma}$ will be a real point. We shall denote this real point by $P_{k}$, and the locus of $P_{k}$ will be a surface $S_{k}$, whose properties we propose to investigate. Similarly we shall study the surface $S_{v}$, locus of $P_{v}$, where $P_{v}$ is the harmonic conjugate of $P_{w}$ with respect to $P_{f}$ and $P_{g}$.

It follows from (14) that the homogeneous coördinates of $P_{k}$ are given by

We find

and

$$
k=f+g \text {. }
$$

$$
\frac{\partial k}{\partial z}=m g+\frac{\partial g}{\partial z}, \quad \frac{\partial k}{\partial z_{0}}=n f+\frac{\partial f}{\partial z_{0}},
$$

$$
\begin{gathered}
\frac{\partial^{2} k}{\partial z^{2}}=2 m_{z} g+2 m g_{z}, \quad \frac{\partial^{2} k}{\partial z_{0}^{2}}=2 n_{z_{0}} f+2 n f_{z_{0}}, \\
\frac{\partial^{2} k}{\partial z \partial z_{0}}=\left(m_{z_{0}}+m n\right) g+\left(n_{z}+m n\right) f,
\end{gathered}
$$

where we have written

$$
m_{z}=\frac{\partial m}{\partial z}, \quad n_{z}=\frac{\partial n}{\partial z}, \quad \text { etc. }
$$

But, according to (17), we have

so that we find

$$
m_{z_{0}}=m n, \quad n_{z}=m n,
$$

$$
\frac{\partial^{2} k}{\partial z \partial z_{0}}=2 m n k .
$$

Consequently, the curves on $S_{k}$, which correspond to the null-lines of the plane, form a conjugate system with equal Laplace-Darboux invariants.*

Moreover, the curves of this conjugate system $S_{k}$ are plane curves. $\dagger$ For we have

$$
\frac{\partial^{3} k}{\partial z^{3}}=2\left(m_{z z}+m m_{z}\right) g+2\left(2 m_{z}+m^{2}\right) g_{z} .
$$

From this equation, and the equations for $\partial k / \partial z$ and $\partial^{2} k / \partial z^{2}$, we may eliminate $g$ and $g_{z}$, obtaining a linear homogeneous differential equation of the third order for the curve $z_{0}=$ const. on $S_{k}$, viz.;

$$
\left(m^{2}-m_{z}\right) \frac{\partial^{3} k}{\partial z^{3}}+\left(m_{z z}-m m_{z}-m^{3}\right) \frac{\partial^{2} k}{\partial z^{2}}-2\left(m m_{z z}-2 m_{z}^{2}\right) \frac{\partial k}{\partial z}=0 .
$$

Only if $m_{z}=m^{2}$ will this plane curve reduce to a straight line. But we

* G. Darboux, Théorie des surfaces, vol. 2, p. 27.

$\dagger$ This remark was first made by one of my students, Dr. Cyril Nelson. 
find, from (13),

$$
m_{z}=m^{2}+\frac{w^{\prime \prime \prime}}{w^{\prime \prime}} m, \quad n_{z_{0}}=n^{2}+\frac{w_{0}^{\prime \prime \prime}}{w_{0}^{\prime \prime}} n .
$$

Therefore, if $m_{z}=m^{2}$, we must have either $m=0$ or $w^{\prime \prime \prime}=0$. The former case corresponds to $w=\alpha z+\beta$, where $\alpha$ and $\beta$ are constants, and in this case $S_{k}$ degenerates into a plane. If $m_{z}=m^{2}, m \neq 0$, we have $w^{\prime \prime \prime}=0$, or $w=\alpha z^{2}+\beta z+\gamma$, where $\alpha, \beta$, and $\gamma$ are constants, and $n_{z_{0}}=n^{2}$. Consequently we find, in this case,

$$
k_{z z}=2 m k_{z}, \quad k_{z_{0} z_{0}}=2 n k_{z_{0}}, \quad k_{z z_{0}}=2 m n k,
$$

so that, in this case also, $S_{k}$ degenerates into a plane, ${ }^{*}$ and the curves $z=$ const. and $z_{0}=$ const. in this plane are straight lines.

If we discard these cases, each of the curves $z=$ const. and $z_{0}=$ const. of $S_{k}$, which intersect at $P_{k}$, determine a plane. The plane of the curve $z_{0}=$ const. is determined by

$$
k=f+g, \quad \frac{\partial k}{\partial z}=m g+g_{z}, \quad \frac{\partial^{2} k}{\partial z^{2}}=2 m_{z} g+2 m g_{z} ;
$$

that of the curve $z=$ const. by

$$
k=f+g, \quad \frac{\partial k}{\partial z_{0}}=n f+f_{z_{0}}, \quad \frac{\partial^{2} k}{\partial z_{0}^{2}}=2 n_{z_{0}} f+2 n f_{z_{0}} .
$$

Of course $k=f+g$ is a point of both planes. Since we may write

$$
k_{z z}-2 m k_{z}=2\left(m_{z}-m^{2}\right) g
$$

we see that the point $P_{g}$ is common to both planes, and therefore the same thing is true of $P_{f}$.

In other words; the lines of our congruence are defined completely if the real surface $S_{k}$ and the conjugate system of plane curves on $S_{k}$ is known. They are the lines of intersection of the planes of these curves. Our congruence is the so-called axis congruence of this conjugate system on $S_{k}+\dagger$ It is clear, moreover, that the planes of the curves on $S_{k}$ which form the conjugate system are the tangent planes of the two focal cones.

In order to obtain a complete projective theory of the surface $S_{k}$, we must find the two second order partial differential equations which $k_{1}, k_{2}, k_{3}, k_{4}$ will satisfy. One of these is (30). The other one may be obtained from (34)

\footnotetext{
${ }^{*}$ E. J. Wilczynski, One-parameter family of nets of plane curves. These $\mathrm{T} \mathrm{r}$ a $\mathrm{n}$ s a c $\mathrm{t}$ i o $\mathrm{n}$ s, vol. 12 (1911), p. 474.

$\dagger$ E. J. Wilczynski, The general theory of congruences, These Trans a c tions vol. 16, (1915), pp. 312-317.
} 
and (35) by eliminating $g$. It then appears in the form

$$
\begin{aligned}
\left(n_{z_{0}}-n^{2}\right)\left(k_{z z}-2 m k_{z}\right)+\left(m_{z}-m^{2}\right)\left(k_{z_{0} z_{0}}-2 n k_{z_{0}}\right) & \\
& =2\left(m_{z}-m^{2}\right)\left(n_{z_{0}}-n^{2}\right) k .
\end{aligned}
$$

But, on account of (13) and (33), this becomes

(37) $w_{0}^{\prime \prime \prime} k_{z z}-w^{\prime \prime \prime} k_{z_{0} z_{0}}+\frac{2 w^{\prime \prime} w_{0}^{\prime \prime \prime}}{w^{\prime}-w_{0}^{\prime}} k_{z}+\frac{2 w^{\prime \prime \prime} w_{0}^{\prime \prime}}{w^{\prime}-w_{0}^{\prime}} k_{z_{0}}+\frac{2 w^{\prime \prime \prime} w_{0}^{\prime \prime}}{w^{\prime}-w_{0}^{\prime}} k=0$,

where we shall assume $w^{\prime}-w_{0}^{\prime} \neq 0, w^{\prime \prime \prime} \neq 0$.

This equation shows that the coefficients of the second fundamental form of $S_{k}$, with respect to the parameters $z$ and $z_{0}$, are proportional to

$$
w^{\prime \prime \prime}, \quad 0, \quad w_{0}^{\prime \prime \prime},
$$

so that the differential equation of the asymptotic lines of $S_{k}$ is

$$
w^{\prime \prime \prime} d z^{2}+w_{0}^{\prime \prime \prime} d z_{0}^{2}=0 .
$$

Consequently the conjugate system formed on $S_{k}$ by the curves $z=$ const. and $z_{0}=$ const. is an isothermally conjugate system. The asymptotic lines of $S_{k}$ are obtained by equating to constants the variables

$R=\frac{1}{2}\left[\int \sqrt{w^{\prime \prime \prime}} d z+i \int \sqrt{w_{0}^{\prime \prime \prime}} d z_{0}\right], \quad S=\frac{1}{2}\left[\int \sqrt{w^{\prime \prime \prime}} d z-i \int \sqrt{w_{0}^{\prime \prime \prime}} d z_{0}\right]$.

These variables are not real, but we shall show that they differ from real variables only by constant imaginary factors. For, if we put

$$
\int \sqrt{w^{\prime \prime \prime}} d z=\lambda+i \mu, \quad \int \sqrt{w_{0}^{\prime \prime \prime}} d z_{0}=\lambda-i \mu,
$$

where $\lambda$ and $\mu$ are real, we find

$$
R=\frac{1+i}{2}(\lambda+\mu), \quad S=\frac{1-i}{2}(\lambda-\mu) .
$$

Consequently the variables

$$
r=\frac{1-i}{\sqrt{2}} R, \quad s=-\frac{1+i}{\sqrt{2}} S
$$

are real and constant along the asymptotic lines of $S_{k}$. We find

$$
\begin{aligned}
p & =r+i s \\
& =\frac{1}{2 \sqrt{2}}\left[(1-i) \int \sqrt{w^{\prime \prime \prime}} d z+(1+i) \int \sqrt{w_{0}^{\prime \prime \prime}} d z_{0}\right. \\
& \left.-i(1+i) \int \sqrt{\dot{w}^{\prime \prime \prime}} d z-(1+i) \int \sqrt{w_{0}^{\prime \prime \prime}} d z_{0}\right],
\end{aligned}
$$


or

$$
p=\frac{1-i}{\sqrt{2}} \int \sqrt{w^{\prime \prime \prime}} d z, \quad p_{0}=\frac{1+i}{\sqrt{2}} \int \sqrt{w_{0}^{\prime \prime \prime}} d z_{0},
$$

or, if we prefer,

$$
p=\int \sqrt{i w^{\prime \prime \prime}} d z, \quad p_{0}=\int \sqrt{-i w_{0}^{\prime \prime \prime}} d z_{0} .
$$

Since $r$ and $s$ are the components of the complex variable $p=r+i s$, which is a function of $z=x+i y$, we see that the asymptotic lines of $S_{k}$ correspond to an orthogonal isothermal system of curves in the z-plane, namely to the curves obtained by equating to constants the real and the imaginary parts of the function

$$
p=\frac{1-i}{\sqrt{2}} \int \sqrt{w^{\prime \prime \prime}} d z .
$$

Thus the asymptotic curves on $S_{k}$ are real, and $S_{k}$ is a surface of negative curvature.

If we introduce $p$ and $p_{0}$ as independent variables, the two differential equations for $k$ assume the form

$$
\begin{aligned}
\frac{\partial^{2} k}{\partial p^{2}}+\frac{\partial^{2} k}{\partial p_{0}^{2}}+\frac{1+i}{\sqrt{2}} \frac{1}{\sqrt{w^{\prime \prime \prime}}}\left[\frac{2 w^{\prime \prime}}{w^{\prime}-w_{0}^{\prime}}+\frac{1}{2} \frac{w^{(4)}}{w^{\prime \prime \prime}}\right] \frac{\partial k}{\partial p} \\
-\frac{1-i}{\sqrt{2}} \frac{1}{\sqrt{w_{0}^{\prime \prime}}}\left[\frac{2 w_{0}^{\prime \prime}}{w^{\prime}-w_{0}^{\prime}}-\frac{1}{2} \frac{w_{0}^{(4)}}{w_{0}^{\prime \prime \prime}}\right] \frac{\partial k}{\partial p_{0}}+\frac{2 i}{w^{\prime}-w_{0}^{\prime}} k=0, \\
\frac{\partial^{2} k}{\partial p \partial p_{0}}+\frac{2 w^{\prime \prime} w_{0}^{\prime \prime} k}{\left(w^{\prime}-w_{0}^{\prime}\right)^{2} \sqrt{w^{\prime \prime \prime} w_{0}^{\prime \prime \prime}}}=0
\end{aligned}
$$

Since $p=r+i s, p_{0}=r-i s$, we obtain from this the differential equations of $S_{k}$ referred to its asymptotic lines, namely

where

$$
\begin{aligned}
& \frac{\partial^{2} k}{\partial r^{2}}+\frac{1}{2} \frac{W_{r}}{W} \frac{\partial k}{\partial r}-\frac{1}{2} \frac{W_{s}}{W} \frac{\partial k}{\partial s}+\left[\frac{4 w^{\prime \prime} w_{0}^{\prime \prime}}{W}+\frac{2 i}{w^{\prime}-w_{0}^{\prime}}\right] k=0, \\
& \frac{\partial^{2} k}{\partial s^{2}}-\frac{1}{2} \frac{W_{r}}{W} \frac{\partial k}{\partial r}+\frac{1}{2} \frac{W_{s}}{W} \frac{\partial k}{\partial s}+\left[\frac{4 w^{\prime \prime} w_{0}^{\prime \prime}}{W}-\frac{2 i}{w^{\prime}-w_{0}^{\prime}}\right] k=0,
\end{aligned}
$$

$$
W=\left(w^{\prime}-w_{0}^{\prime}\right)^{2} \sqrt{w^{\prime \prime \prime} w_{0}^{\prime \prime \prime}} \text {. }
$$

We have already observed that we obtain a second real surface $S_{v}$ as the locus of $P_{v}$ where $P_{v}$ is the harmonic conjugate of $P_{w}$ with respect to $P_{f}$ and $P_{g}$. Since the homogeneous coördinates of $P_{w}$ are given by $\mu=w_{0}^{\prime} f-w^{\prime} g$, those of $P_{v}$ will be

We find

$$
v=w_{0}^{\prime} f+w^{\prime} g .
$$

$$
\begin{gathered}
\frac{\partial v}{\partial z}=\left(w_{0}^{\prime} m+w^{\prime \prime}\right) g+w^{\prime} g_{z} \\
\frac{\partial^{2} v}{\partial z \partial z_{0}}=\left(w_{0}^{\prime \prime} m+w_{0}^{\prime} m n+w^{\prime} m n\right) g+\left(w^{\prime \prime} n+w_{0}^{\prime} m n+w^{\prime} m n\right) f
\end{gathered}
$$


which reduces to

$$
\frac{\partial^{2} v}{\partial z \partial z_{0}}=2 m n v
$$

Of course the properties of $S_{v}$, in relation to our congruence, are essentially the same as those of $S_{k}$, but equation (43) shows further that $S_{k}$ and $S_{v}$ satisfy the same Moutard equation.

\section{The method of the RiemanN sphere}

We now pass to the second method mentioned in the introduction. We have, as before,

$$
w=u+i v=F(z)=F(x+i y) .
$$

We use the $\xi \eta$-plane as the plane of both of the complex variables. Let $P_{z}$ and $P_{w}$ be two points of the $\xi \eta$-plane which represent a value of $z$ and the corresponding value of $w$, and let $P_{1}$ and $P_{2}$ be the two points obtained from $P_{z}$ and $P_{w}$, upon the sphere of unit radius and center at the origin, by stereographic projection from the point $(0,0,1)$. The coördinates of $P_{1}$ and $P_{2}$ will be

and

$$
\xi_{1}=\frac{z+z_{0}}{z z_{0}+1}, \quad \eta_{1}=\frac{-i\left(z-z_{0}\right)}{z z_{0}+1}, \quad \zeta_{1}=\frac{z z_{0}-1}{z z_{0}+1}
$$

$$
\xi_{2}=\frac{w+w_{0}}{w w_{0}+1}, \quad \eta_{2}=\frac{-i\left(w-w_{0}\right)}{w w_{0}+1}, \quad \zeta_{2}=\frac{w w_{0}-1}{w w_{0}+1}
$$

respectively. We may therefore write for the homogeneous cartesian coordinates of $P_{1}$,

$$
\lambda_{1}=z+z_{0}, \quad \lambda_{2}=-i\left(z-z_{0}\right), \quad \lambda_{3}=z z_{0}-1, \quad \lambda_{4}=z z_{0}+1,
$$

and for those of $P_{2}$,

(45) $\mu_{1}=w+w_{0}, \quad \mu_{2}=-i\left(w-w_{0}\right), \quad \mu_{3}=w w_{0}-1, \quad \mu_{4}=w w_{0}+1$,

where, of course,

$$
w=F(z), \quad w_{0}=F_{0}\left(z_{0}\right) .
$$

We propose to study the congruence generated by the line $P_{1} P_{2}$ when $z=x+i y$ ranges over the domain in which the function $F(z)$ is defined. In order to apply the general theory of congruences we must find two linear partial differential equations of the first order, and two of the second order which are satisfied by the four pairs of functions $\left(\lambda_{k}, \mu_{k}\right)$. Since $\lambda_{k}$ is linear in $z$, and $\mu_{k}$ is linear in $w$, the second order equations may be taken in the form

$$
\frac{\partial^{2} \lambda}{\partial z^{2}}=0, \quad \frac{\partial^{2} \mu}{\partial z^{2}}-\frac{w^{\prime \prime}}{w^{\prime}} \frac{\partial \mu}{\partial z}=0
$$


the latter being derived from

$$
\frac{\partial^{2} \mu}{\partial w^{2}}=0
$$

by introducing $z$ as independent variable. Of course we have also

$$
\frac{\partial^{2} \lambda}{\partial z_{0}^{2}}=0, \quad \frac{\partial^{2} \mu}{\partial z_{0}^{2}}-\frac{w_{0}^{\prime \prime}}{w_{0}^{\prime}} \frac{\partial \mu}{\partial z_{0}}=0,
$$

but these are consequences of (46) and the two first order equations which are still to be found. To obtain these we use the method of undetermined coefficients. We write

$$
\begin{aligned}
& \frac{\partial \lambda}{\partial z_{0}}=a_{11} \frac{\partial \lambda}{\partial z}+a_{12} \frac{\partial \mu}{\partial z}+b_{11} \lambda+b_{12} \mu, \\
& \frac{\partial \mu}{\partial z_{0}}=a_{21} \frac{\partial \lambda}{\partial z}+a_{22} \frac{\partial \mu}{\partial z}+b_{21} \lambda+b_{22} \mu,
\end{aligned}
$$

and determine the coefficients $a_{i k}$ and $b_{i k}$ by demanding that each of the equations (48) shall be satisfied when we put

We find

$$
\lambda=\lambda_{k}, \quad \mu=\mu_{k}, \quad(k=1,2,3,4) .
$$

$$
a_{11}=0, \quad a_{12}=-\frac{z-w}{z_{0}-w_{0}} \frac{d z}{d w}, \quad b_{11}=-b_{12}=\frac{1}{z_{0}-w_{0}},
$$

$$
a_{21}=-\frac{z-w}{z_{0}-w_{0}} \frac{d w_{0}}{d z_{0}}, \quad a_{22}=\dot{0}, \quad b_{21}=-b_{22}=\frac{\frac{d w_{0}}{d z_{0}}}{z_{0}-w_{0}} .
$$

Thus, the desired system of partial differential equations is the following:

$$
\begin{gathered}
\frac{\partial \lambda}{\partial z_{0}}=-\frac{z-w}{z_{0}-w_{0}} \frac{d z}{d w} \frac{\partial \mu}{\partial z}+\frac{1}{z_{0}-w_{0}}(\lambda-\mu), \\
\frac{\partial \mu}{\partial z_{0}}=-\frac{z-w}{z_{0}-w_{0}} \frac{d w_{0}}{d z_{0}} \frac{\partial \lambda}{\partial z}+\frac{\frac{d w_{0}}{d z_{0}}}{z_{0}-w_{0}}(\lambda-\mu), \\
\frac{\partial^{2} \lambda}{\partial z^{2}}=0, \quad \frac{\partial^{2} \mu}{\partial z^{2}}-\frac{w^{\prime \prime}}{w^{\prime}} \frac{\partial \mu}{\partial z}=0 .
\end{gathered}
$$

Equations (46) show that the ruled surfaces $z_{0}=$ const. of the congruence have two straight-line directrices. That this must be so is, of course, evident. For, on account of the conformal character of the correspondence between $z$ and $w$, and since the stereographic projection is also conformal, $z$ and $w$ will both describe null-lines of the $\xi \eta$-plane if either of them does, and then 
the points $P_{1}$ and $P_{2}$ will simultaneously describe null-lines (imaginary generators) of the sphere.

The foci of the line $P_{1} P_{2}$, that is the two points where the line touches the focal surface of the congruence, may be obtained by means of general methods familiar to geometers. As applied to a system of form (48), these methods show that the homogeneous coördinates of the foci are the factors of the quadratic covariant

$$
a_{21} \lambda^{2}-\left(a_{11}-a_{22}\right) \lambda \mu-a_{12} \mu^{2} .
$$

If we use (49), we find that, in our case, the foci are given by

where

$$
\bar{\lambda}=\mu+\sqrt{w^{\prime} w_{0}^{\prime}} \lambda, \quad \bar{\mu}=\mu-\sqrt{w^{\prime} w_{0}^{\prime}} \lambda,
$$

$$
w^{\prime}=\frac{d w}{d z}=F^{\prime}(z), \quad w_{0}^{\prime}=\frac{d w_{0}}{d z_{0}}=F_{0}^{\prime}\left(z_{0}\right) .
$$

Since $w^{\prime} w_{0}^{\prime}$ is positive, and since $\lambda_{k}$ and $\mu_{k}$ are real, we see that the focal surfaces of this congruence are always real. Moreover, equations (51) show, that the foci of every line of the congruence separate harmonically the two points, $P_{1}$ and $P_{2}$, in which this line intersects the Riemann sphere. Finally we observe that the two foci of a line are distinct except upon those lines of the congruence which correspond to a solution of the equation $F^{\prime}(z)=0$ or of $F^{\prime}(z)=\infty$.

Thus the two sheets of the focal surface can hang together only at those points for which $F^{\prime}(z)$ becomes zero or infinite.

The developables of the congruence may be obtained, in the general case of a system of form (48), by equating to constants two independent solutions, $\varphi\left(z, z_{0}\right)$ and $\psi\left(z, z_{0}\right)$, of the partial differential equation

$$
\left(\frac{\partial \theta}{\partial z_{0}}\right)^{2}-\left(a_{11}+a_{22}\right) \frac{\partial \theta}{\partial z} \frac{\partial \theta}{\partial z_{0}}+\left(a_{11} a_{22}-a_{12} a_{21}\right)\left(\frac{\partial \theta}{\partial z}\right)^{2}=0 . *
$$

In our case, using the values of $a_{i k}$ given by (49), it is easy to perform the integration. We find in this way the following result.

The developables of our congruence are the ruled surfaces given by $\alpha=$ const. and $\beta=$ const., where

$$
\alpha+i \beta=t=i \int \frac{\sqrt{w^{\prime} d z}}{z-w}, \quad \alpha-\imath \beta=\tau_{0}=-i \int \frac{\sqrt{w_{0}^{\prime}} d z_{0}}{z_{0}-w_{0}} .
$$

Since $\alpha$ and $\beta$ are the components of a complex variable $t$ which is a function of $z$ alone, the developables of the congruence correspond to an orthogonal isothermal system of curves on the Riemann sphere.

${ }^{*}$ Here, as well as in the determination of the focal surfaces, I am making use of some formulæ from a theory of one-parameter families of ruled surfaces which is, as yet, unpublished. The reader, who finds it difficult to prove these general formulæ, should follow the transformations mechanically. The final results enable him to verify, a posteriori, the correctness of our statements. 
We shall have to transform equations (50) into an equivalent system for the functions $\bar{\lambda}$ and $\bar{\mu}$ of $\alpha$ and $\beta$, before we can apply the formulæ of the general theory of congruences to our particular case. This transformation may be accomplished as follows.

From (51), we find

$$
\lambda=\frac{1}{2 \sqrt{w^{\prime} w_{0}^{\prime}}}(\bar{\lambda}-\bar{\mu}), \quad \mu=\frac{1}{2}(\bar{\lambda}+\bar{\mu}) .
$$

If we substitute these values in (50), we find

$$
\begin{aligned}
& \frac{\partial \bar{\lambda}}{\partial z_{0}}=-\sqrt{\frac{w_{0}^{\prime}}{w^{\prime}}} \frac{z-w}{z_{0}-w_{0}} \frac{\partial \bar{\lambda}}{\partial z}+\frac{1}{2\left(z_{0}-w_{0}\right)}\left(1+\sqrt{\frac{w_{0}^{\prime}}{w^{\prime}}}\right)[\bar{\lambda}-\bar{\mu} \\
& \left.-\sqrt{w^{\prime} w_{0}^{\prime}}(\bar{\lambda}+\bar{\mu})\right]+\frac{1}{4}\left[\frac{w_{0}^{\prime \prime}}{w_{0}^{\prime}}+\frac{z-w}{z_{0}-w_{0}} \sqrt{\frac{w_{0}^{\prime}}{w^{\prime}}} \frac{w^{\prime \prime}}{w^{\prime}}\right](\bar{\lambda}-\bar{\mu}), \\
& \frac{\partial \bar{\mu}}{\partial z_{0}}=+\sqrt{\frac{w_{0}^{\prime}}{w^{\prime}} \frac{z-w}{z_{0}-w_{0}}} \frac{\partial \bar{\mu}}{\partial z}-\frac{1}{2\left(z_{0}-w_{0}\right)}\left(1-\sqrt{\frac{w_{0}^{\prime}}{w^{\prime}}}\right)[\bar{\lambda}-\bar{\mu} \\
& \left.-\sqrt{w^{\prime} w_{0}^{\prime}}(\bar{\lambda}+\bar{\mu})\right]-\frac{1}{4}\left[\frac{w_{0}^{\prime \prime}}{w_{0}^{\prime}}-\frac{z-w}{z_{0}-w_{0}} \sqrt{\frac{w_{0}^{\prime}}{w^{\prime}}} \frac{w^{\prime \prime}}{w^{\prime}}\right](\bar{\lambda}-\bar{\mu}),
\end{aligned}
$$

and

$$
\begin{aligned}
& \frac{\partial^{2} \bar{\lambda}}{\partial z^{2}}-\frac{w^{\prime \prime}}{w^{\prime}} \frac{\partial \bar{\lambda}}{\partial z}-\frac{1}{4}\{w, z\}(\bar{\lambda}-\bar{\mu})=0, \\
& \frac{\partial^{2} \bar{\mu}}{\partial z^{2}}-\frac{w^{\prime \prime}}{w^{\prime}} \frac{\partial \bar{\mu}}{\partial z}+\frac{1}{4}\{w, z\}(\bar{\lambda}-\bar{\mu})=0,
\end{aligned}
$$

where $\{w, z\}$ is the Schwarzian derivative of $w$ with respect to $z$, and where a definite determination must be chosen in every specific case of a branch of the function $\sqrt{w^{\prime}}$; the value of $\sqrt{w_{0}^{\prime}}$ will then be uniquely determined if we agree that $\sqrt{w^{\prime} w_{0}^{\prime}}=\sqrt{w^{\prime}} \sqrt{w_{0}^{\prime}}$ shall mean the positive value of this square root.

We continue the transformation by introducing the variables, $t$ and $t_{0}$, defined by (52), in place of $z$ and $z_{0}$. The result of this transformation on equations (54) is

$$
\begin{aligned}
-i \sqrt{w^{\prime} w_{0}^{\prime}}\left(\frac{\partial \bar{\lambda}}{\partial t_{0}}-\frac{\partial \bar{\lambda}}{\partial t}\right) & \\
= & +\left[\frac{1}{2}\left(\sqrt{w^{\prime}}+\sqrt{w_{0}^{\prime}}\right)\left(1-\sqrt{w^{\prime} w_{0}^{\prime}}\right)\right. \\
+ & \left.\frac{1}{4}\left\{\left(z_{0}-w_{0}\right) \sqrt{w^{\prime}} \frac{w_{0}^{\prime \prime}}{w_{0}^{\prime}}+(z-w) \sqrt{w_{0}^{\prime}} \frac{w^{\prime \prime}}{w^{\prime}}\right\}\right] \bar{\lambda} \\
& -\left[\frac{1}{2}\left(\sqrt{w^{\prime}}+\sqrt{w_{0}^{\prime}}\right)\left(1+\sqrt{w^{\prime} w_{0}^{\prime}}\right)\right.
\end{aligned}
$$




$$
\begin{aligned}
+i \sqrt{w^{\prime} w_{0}^{\prime}}\left(\frac{\partial \bar{\mu}}{\partial t_{0}}+\frac{\partial \bar{\mu}}{\partial t}\right) & \left.\frac{1}{4}\left\{\left(z_{0}-w_{0}\right) \sqrt{w^{\prime}} \frac{w_{0}^{\prime \prime}}{w_{0}^{\prime}}+(z-w) \sqrt{w_{0}^{\prime}} \frac{w^{\prime \prime}}{w^{\prime}}\right\}\right] \bar{\mu}, \\
= & -\left[\frac{1}{2}\left(\sqrt{w^{\prime}}-\sqrt{w_{0}^{\prime}}\right)\left(1-\sqrt{w^{\prime} w_{0}^{\prime}}\right)\right. \\
+ & \left.\frac{1}{4}\left\{\left(z_{0}-w_{0}\right) \sqrt{w^{\prime}} \frac{w_{0}^{\prime \prime}}{w_{0}^{\prime}}-(z-w) \sqrt{w_{0}^{\prime}} \frac{w^{\prime \prime}}{w^{\prime}}\right\}\right] \bar{\lambda} \\
+ & {\left[\frac{1}{2}\left(\sqrt{w^{\prime}}-\sqrt{w_{0}^{\prime}}\right)\left(1+\sqrt{w^{\prime} w_{0}^{\prime}}\right)\right.} \\
+ & \left.\frac{1}{4}\left\{\left(z_{0}-w_{0}\right) \sqrt{w^{\prime}} \frac{w_{0}^{\prime \prime}}{w_{0}^{\prime}}-(z-w) \sqrt{w_{0}^{\prime}} \frac{w^{\prime \prime}}{w^{\prime}}\right\}\right] \bar{\mu} .
\end{aligned}
$$

It will not be necessary to apply this transformation to (55) just now; we shall do this presently however in a slightly modified form.

Since $t=\alpha+i \beta, t_{0}=\alpha-i \beta$, we obtain, from (56), a system of the form

$$
\frac{\partial \bar{\lambda}}{\partial \beta}=m^{\prime} \bar{\lambda}+m^{\prime \prime} \bar{\mu}, \quad \frac{\partial \bar{\mu}}{\partial \alpha}=n^{\prime} \bar{\lambda}+n^{\prime \prime} \bar{\mu},
$$

where

$$
\begin{aligned}
& 4 m^{\prime}=2\left(\frac{1}{\sqrt{w^{\prime}}}+\frac{1}{\sqrt{w_{0}^{\prime}}}\right)\left(1-\sqrt{w^{\prime} w_{0}^{\prime}}\right) \\
& +\left(z_{0}-w_{0}\right) \frac{w_{0}^{\prime \prime}}{\left(w_{0}^{\prime}\right)^{3 / 2}}+(z-w) \frac{w^{\prime \prime}}{\left(w^{\prime}\right)^{3 / 2}}, \\
& 4 m^{\prime \prime}=-2\left(\frac{1}{\sqrt{w^{\prime}}}+\frac{1}{\sqrt{w_{0}^{\prime}}}\right)\left(1+\sqrt{w^{\prime} w_{0}^{\prime}}\right) \\
& -\left(z_{0}-w_{0}\right) \frac{w_{0}^{\prime \prime}}{\left(w_{0}^{\prime}\right)^{3 / 2}}-(z-w) \frac{w^{\prime \prime}}{\left(w^{\prime}\right)^{3 / 2}}, \\
& 4 i n^{\prime}=2\left(\frac{1}{\sqrt{w_{0}}}-\frac{1}{\sqrt{w^{\prime}}}\right)\left(1-\sqrt{w^{\prime} w_{0}^{\prime}}\right) \\
& +\left(z_{0^{\prime}}-w_{0}\right) \frac{w_{0}^{\prime \prime}}{\left(w_{0}^{\prime}\right)^{3 / 2}}-(z-w) \frac{. u^{\prime \prime}}{\left(w^{\prime}\right)^{3 / 2}}, \\
& 4 i n^{\prime \prime}=-2\left(\frac{1}{\sqrt{w_{0}^{\prime}}}-\frac{1}{\sqrt{w^{\prime}}}\right)\left(1+\sqrt{w^{\prime} w_{0}^{\prime}}\right) \\
& -\left(z_{0}-w_{0}\right) \frac{w_{0}^{\prime \prime}}{\left(w_{0}^{\prime}\right)^{3 / 2}}+(z-w) \frac{w^{\prime \prime}}{\left(w^{\prime}\right)^{3 / 2}} .
\end{aligned}
$$

A transformation of the form

$$
\bar{\lambda}=\rho f, \quad \bar{\mu}=\sigma g
$$

can always be made so as to transform a system of form (57) into one of the binomial form

Trans. Am. Math. Soc. 20

$$
\frac{\partial f}{\partial \beta}=m g, \quad \frac{\partial g}{\partial \alpha}=n f,
$$


by imposing the conditions

$$
\frac{\partial \rho}{\partial \beta}=m^{\prime} \rho, \quad \frac{\partial \sigma}{\partial \alpha}=n^{\prime \prime} \sigma
$$

upon $\rho$ and $\sigma$. We can satisfy these conditions in our case by putting

$$
\rho=\sigma=\sqrt[4]{w^{\prime} w_{0}^{\prime}} \sqrt{(z-w)\left(z_{0}-w_{0}\right)},
$$

so that we may even choose $\rho$ and $\sigma$ as positive quantities. Consequently the transformation

$$
\begin{aligned}
& f=\frac{\bar{\lambda}}{\sqrt[4]{w^{\prime} w_{0}^{\prime}} \sqrt{(z-w)\left(z_{0}-w_{0}\right)}}=\frac{\sqrt[4]{w^{\prime} w_{0}^{\prime}} \lambda+\frac{\mu}{\sqrt[4]{w^{\prime} w_{0}^{\prime}}}}{\sqrt{(z-w)\left(z_{0}-w_{0}\right)}}, \\
& g=\frac{-\sqrt[4]{w^{\prime} w_{0}^{\prime}} \lambda+\frac{\mu}{\sqrt[4]{w^{\prime} w_{0}^{\prime}}}}{\sqrt[4]{w^{\prime} w_{0}} \sqrt{(z-w)\left(z_{0}-w_{0}\right)}}=\frac{-}{\sqrt{(z-w)\left(z_{0}-w_{0}\right)}},
\end{aligned}
$$

furnishes the first order equations of our system in their final form

$$
f_{\beta}=m g, \quad g_{a}=n f,
$$

where $f_{\beta}=\partial f / \partial \beta$, etc., and where $m$ and $n$, which are real functions of $\alpha$ and $\beta$, are defined by

$$
m+i n=-\frac{1}{\sqrt{w^{\prime}}}\left[1+w^{\prime}+\frac{1}{2}(z-w) \frac{w^{\prime \prime}}{w^{\prime}}\right]
$$

whence

$$
m-i n=-\frac{1}{\sqrt{w_{0}^{\prime}}}\left[1+w_{0}^{\prime}+\frac{1}{2}\left(z_{0}-w_{0}\right) \frac{w_{0}^{\prime \prime}}{w_{0}^{\prime}}\right] \text {. }
$$

By means of (60) we have four pairs of functions $\left(f_{k}, g_{k}\right)$ corresponding to the four pairs $\left(\lambda_{k}, \mu_{k}\right)$. Since the coördinates, which we are using, are homogeneous the points $P_{f}$ and $P_{g}$ coincide with the foci $P_{\bar{\lambda}}$ and $P_{\bar{\mu}}$ respectively.

In order to complete the transformation of our second order equations, we first use (60) to express $\bar{\lambda}$ and $\bar{\mu}$ in terms of $f$ and $g$. The result of the substitution of these values in (55) is

$$
\begin{aligned}
& \frac{\partial^{2} f}{\partial z^{2}}-\frac{t^{\prime \prime}}{t^{\prime}} \frac{\partial f}{\partial z}-\frac{1}{4}\left[\left(\frac{w^{\prime \prime}}{w^{\prime}}-\frac{t^{\prime \prime}}{t^{\prime}}\right)^{2}+\frac{2 w^{\prime \prime}}{z-w}\right] f+\frac{1}{4}\{w, z\} g=0, \\
& \frac{\partial^{2} g}{\partial z^{2}}-\frac{t^{\prime \prime}}{t^{\prime}} \frac{\partial g}{\partial z}+\frac{1}{4}\{w, z\} f-\frac{1}{4}\left[\left(\frac{w^{\prime \prime}}{w^{\prime}}-\frac{t^{\prime \prime}}{t^{\prime}}\right)^{2}+\frac{2 w^{\prime \prime}}{z-w}\right] g=0,
\end{aligned}
$$

whence

$$
\begin{gathered}
\left.\frac{\partial^{2} f}{\partial t^{2}}-\frac{1}{4\left(t^{\prime}\right)^{2}}\left[\left(\frac{w^{\prime \prime}}{w^{\prime}}-\frac{t^{\prime \prime}}{t^{\prime}}\right)^{2}+\frac{2 w^{\prime \prime}}{z-w}\right)\right] f+\frac{\{w, z\}}{4\left(t^{\prime}\right)^{2}} g=0, \\
\frac{\partial^{2} g}{\partial t^{2}}+\frac{\{w, z\}}{4\left(t^{\prime}\right)^{2}} f-\frac{1}{4\left(t^{\prime}\right)^{2}}\left[\left(\frac{w^{\prime \prime}}{w^{\prime}}-\frac{t^{\prime \prime}}{t^{\prime}}\right)^{2}+\frac{2 w^{\prime \prime}}{z-w}\right] g=0
\end{gathered}
$$


where

$$
\begin{gathered}
t^{\prime}=\frac{d t}{d z}=\frac{i \sqrt{w^{\prime}}}{z-w}, \quad \frac{t^{\prime \prime}}{t^{\prime}}=-\frac{1-w^{\prime}}{z-w}+\frac{1}{2} \frac{w^{\prime \prime}}{w^{\prime}}, \\
\frac{w^{\prime \prime}}{w^{\prime}}-\frac{t^{\prime \prime}}{t^{\prime}}=\frac{1-w^{\prime}}{z-w}+\frac{1}{2} \frac{w^{\prime \prime}}{w^{\prime}} .
\end{gathered}
$$

Finally we find, from (65),

$$
\begin{aligned}
& f_{a \alpha}=a f+b g+c f_{\alpha}+d g_{\beta}, \\
& g_{\beta \beta}=a^{\prime} f+b^{\prime} g+c^{\prime} f_{\alpha}+d^{\prime} g_{\beta},
\end{aligned}
$$

where

$$
\begin{array}{rlrl}
a & =4+n^{2}-m^{2}, & b & =\frac{1}{4}\left[\frac{(z-w)^{2}}{w^{\prime}}\{w, z\}+\frac{\left(z_{0}-w_{0}\right)^{2}}{w_{0}^{\prime}}\left\{w_{0}, z_{0}\right\}\right]=-m_{\beta}, \\
c & =0, \quad d=m, \\
a^{\prime}=-b, & b^{\prime}=-a, \quad c^{\prime}=n, \quad d^{\prime}=0 .
\end{array}
$$

Equations (61) and (67) constitute the system of partial differential equations of our congruence, in its canonical form. The second form, given here for the value of $b$, is an immediate consequence of the following relations which are useful in a large number of questions;

$$
\begin{aligned}
& m_{z}=-\frac{1}{4} \frac{z-w}{\sqrt{w^{\prime}}}\{w, z\}, \quad m_{z_{0}}=-\frac{1}{4} \frac{z_{0}-w_{0}}{\sqrt{w_{0}^{\prime}}}\left\{w_{0}, z_{0}\right\}, \\
& n_{z}=\frac{i}{4} \frac{z-w}{\sqrt{w^{\prime}}}\{w, z\}, \quad n_{z_{0}}=-\frac{i}{4} \frac{z_{0}-w_{0}}{\sqrt{w_{0}^{\prime}}}\left\{w_{0}, z_{0}\right\} \text {, } \\
& m_{t}=+\frac{i}{4} \frac{(z-w)^{2}}{w^{\prime}}\{w, z\}, \quad m_{t_{0}}=-\frac{i}{4} \frac{\left(z_{0}-w_{0}\right)^{2}}{w_{0}^{\prime}}\left\{w_{0}, z_{0}\right\} \\
& n_{t}=+\frac{1}{4} \frac{(z-w)^{2}}{w^{\prime}}\{w, z\}, \quad n_{t_{0}}=+\frac{1}{4} \frac{\left(z_{0}-u_{0}\right)^{2}}{w_{0}^{\prime}}\left\{w_{0}, z_{0}\right\}, \\
& m_{a}=\frac{i}{4}\left[\frac{(z-w)^{2}}{w^{\prime}}\{w, z\}-\frac{\left(z_{0}-w_{0}\right)^{2}}{w_{0}^{\prime}}\left\{w_{0}, z_{0}\right\}\right] \text {, } \\
& m_{\beta}=-\frac{1}{4}\left[\frac{(z-w)^{2}}{w^{\prime}}\{w, z\}+\frac{\left(z_{0}-w_{0}\right)^{2}}{w_{0}^{\prime}}\left\{w_{0}, z_{0}\right\}\right] \text {, } \\
& n_{a}=-m_{\beta}, \quad n_{\beta}=m_{a},
\end{aligned}
$$

where the last two equations are merely expressive of the fact, already noted, that $m+i n$ is a function of $x+i y$ and therefore of $\alpha+i \beta$.

The focal sheets of the congruence can degenerate into curves only if $m$ or $n$, or both $m$ and $n$, are equal to zero. Equations (69) show that this can happen 
only if $w$ is a linear function of $z$, say

$$
w=\frac{\alpha z+\beta}{\gamma z+\delta}
$$

where we may assume $\alpha \delta-\beta \gamma=1$, since the case $w=$ const. excluded by this hypothesis, is of no interest. We find from (62), if we choose

$$
\sqrt{w^{\prime}}=(\gamma z+\delta)^{-1}, \quad m+i n=-(\alpha+\delta), \quad m-i n=-\left(\alpha_{0}+\delta_{0}\right),
$$

so that

$$
2 m=-\left(\alpha+\alpha_{0}+\delta+\delta_{0}\right), \quad 2 i n=-\left(\alpha-\alpha_{0}+\delta-\delta_{0}\right) .
$$

Consequently we obtain the following result.

The sheet $S_{f}$ of the focal surface is degenerate if and only if $w$ is a linear function of $z$ of the form

$$
w=\frac{\alpha z+\beta}{\gamma z+\delta}, \quad \alpha \delta-\beta \gamma=1
$$

for which the real parts of $\alpha$ and $\delta$ are numerically equal but opposite in sign. The second sheet $S_{g}$ degenerates if and only if the corresponding relation holds between the imaginary parts of $\alpha$ and $\delta$, that is, if $\alpha$ and $\delta$ are conjugate complex.* Both sheets degenerate if and only if

$$
\alpha+\delta=0 \text {. }
$$

In all other cases, even if $w$ is a linear function of $z, \dagger$ the focal surfaces are non-degenerate. Equations (68) show that

$$
W=m n-c^{\prime} d=0
$$

in all cases. Consequently the congruence is a $W$-congruence, $\ddagger$ that is, it has the property of making the asymptotic lines of the two sheets of the focal surface correspond to each other. That this is so, also follows directly from equations (55). For we may regard (55) as the differential equations of a ruled surface $z_{0}=$ const. of our congruence. The form of the equations shows immediately that the curves of contact of this ruled surface with the two sheets of the focal surface are asymptotic curves upon both of them. $\$$ Of course the same facts may be read off from equations (64) or (65).

These simple remarks enable us to conclude further that the ruled surfaces

* If besides, $\beta$ and $-\gamma$ are conjugate complex, (70) reduces to a rotation of the Riemann sphere.

$\dagger$ If $w$ is a linear function, aside from these exceptional cases, both sheets of the focal surface are quadrics.

$\ddagger$ Brussels paper, p. 34 and p. 46 .

$\S$ E. J. Wilczynski, Projective differential geometry of curves and ruled surfaces, Leipzig (1906), p. 142. 
$z_{0}=$ const. and $z=$ const., that is, the ruled surfaces of the congruence which are determined by the imaginary generators of the Riemann sphere are the ones which touch the focal surface along its asymptotic lines.

Since these ruled surfaces are imaginary, while the focal surface is real, both sheets of the focal surface must have positive curvature everywhere. But the consideration of these ruled surfaces teaches us still more. The curve of contact with either sheet of the focal surface is an asymptotic curve, not only of the focal surface, but of the ruled surface as well. But this ruled surface has two straight line directrices, namely the two imaginary generators of the Riemann sphere which the points $P_{1}$ and $P_{2}$ describe when $z_{0}$ remains constant. Every asymptotic line on a ruled surface with two straight line directrices has the property that each of its asymptotic lines belongs to a linear complex, that is, there exists a linear complex which contains all of its tangents. It follows then that every asymptotic line on each sheet of the focal surface of our congruence belongs to a linear complex.

We wish to verify this conclusion analytically, and also to study some other questions which arise in this connection. If we eliminate $g$ and $g_{\beta}$ from equations (61) and (67) we find

$$
\begin{gathered}
f_{a a}-f_{\beta \beta}=-2 \frac{m_{\beta}}{m} f_{\beta}+a f, \\
f_{a \beta}=\frac{m_{a}}{m} f_{\beta}+m n f,
\end{gathered}
$$

the differential equations of the focal sheet $S_{f}$ referred to the curves $\alpha=$ const. and $\beta=$ const., which are the curves along which the developables of the congruence touch $S_{f}$. The second of these equations merely states the familiar fact that the curves $\alpha=$ const. and $\beta=$ const. form a conjugate system on $S_{f}$; the first equation of (71) shows that this system of curves on $S_{f}$ is isothermally conjugate.

Let us transfrom (71) by introducing $t=\alpha+i \beta$ and $t_{0}=\alpha-i \beta$ as independent variables. We find a system of differential equations of the form

where

$$
\begin{gathered}
\frac{\partial^{2} f}{\partial t^{2}}+2 A \frac{\partial f}{\partial t}+2 B \frac{\partial f}{\partial t_{0}}+C f=0 \\
\frac{\partial^{2} f}{\partial t_{0}^{2}}+2 A^{\prime} \frac{\partial f}{\partial t}+2 B^{\prime} \frac{\partial f}{\partial t_{0}}+C^{\prime} f=0
\end{gathered}
$$

$$
\begin{array}{lll}
A=-\frac{1}{2} \frac{\partial \log m}{\partial t}, & B=+\frac{1}{2} \frac{\partial \log m}{\partial t}, & C=\frac{1}{4}\left[(m+i n)^{2}-4\right] \\
A^{\prime}=+\frac{1}{2} \frac{\partial \log m}{\partial t_{0}}, & B^{\prime}=-\frac{1}{2} \frac{\partial \log m}{\partial t_{0}}, & C^{\prime}=\frac{1}{4}\left[(m-i n)^{2}-4\right]
\end{array}
$$


Equations (72) are the differential equations of the surface $S_{f}$ referred to its asymptotic lines.* From (62), (63), and (73) we find

$$
\frac{\partial^{2} \log A^{\prime}}{\partial t \partial t_{0}}=\frac{\partial^{2} \log B}{\partial t \partial t_{0}}=4 A^{\prime} B
$$

But these equations constitute the analytic verification, which we were seeking; they show, according to C. T. Sullivan, that the asymptotic curves of $S_{f}$ belong to linear complexes. $\dagger$ Sullivan has also noted that every osculating ruled surface of such a surface has two straight-line directrices, and that the locus of these directrices is a quadric surface, which he calls the directrix quadric. $\neq$ It is evident, from what precedes, that in our case the directrix quadric associated in this way with each of the two sheets of the focal surface is the Riemann sphere.

Let us consider an arbitrary point $P$ of the surface $S_{f}$. The osculating linear complexes of the two asymptotic curves of $S_{f}$, which cross at $P$, have a linear congruence in common. The directrices of this congruence are called the directrices of the point $P$; one of them passes through $P$ while the other lies in the plane tangent to $S_{f}$ at $P$. The resulting two congruences have the property that their developables correspond to each other and to a common net of curves on $S_{f}$ called its directrix curves. $\S$ For a system of form (72), the differential equation of the directrix curves is

where

$$
B L d t^{2}+2 M d t d t_{0}-A^{\prime} N d t_{0}^{2}=0,
$$

$$
\begin{aligned}
& L=-2 A^{\prime}\left(2 A^{\prime} B F+2 A^{\prime} B B_{t_{0}}+B A_{t t}^{\prime}\right)+B A_{t}^{\prime 2}, \\
& M=A^{\prime 2} B^{2} \frac{\partial^{2} \log \left(B / A^{\prime}\right)}{\partial t \partial t_{0}}, \\
& N=-2 B\left(2 A^{\prime} B G+2 A^{\prime} B A_{t}^{\prime}+A^{\prime} B_{t_{0} t_{0}}\right)+A^{\prime} B_{t_{0}}^{2} \|
\end{aligned}
$$

In our case this differential equation reduces to

$$
\left[4-(m+i n)^{2}\right] d t^{2}-\left[4-(m-i n)^{2}\right] d t_{0}^{2}=0 .
$$

Consequently the directrix curves on $S_{f}$ form a conjugate system and its equations can be obtained by quadratures. This property however is not peculiar to our case; it is common to all surfaces whose asymptotic lines belong to linear

* E. J. Wilczynski, Projective differential geometry of curved surfaces. (First Memoir), these Trans actions, vol. 8 (1907), p. 244.

†C. T. Sullivan, Properties of surfaces whose asymptotic lines belong to linear complexes, these Trans a ctions, vol. 13 (1912), p. 175.

$\ddagger$ L. c., p. 185.

$\S$ E. J. Wilczynski, Projective differential geometry of curved surfaces (Second Memoir), these Trans a c tion s, vol. 9 (1908), p. 95.

\| L. c., p. 116, equations (136) and (137). 
complexes.* But if we set up the differential equation for the directrix curves on $S_{g}$ we obtain (75) over again. Consequently the directrix curves of the two sheets of the focal surface correspond to each other. This property is not known to be an obvious consequence of the fact that our congruence is a $W$ congruence. On account of this latter property, the system of curves on $S_{\sigma}$ which corresponds to the directrix curves of $S_{f}$ must be a conjugate system, but it need not therefore be composed of the directrix curves of $S_{g}$.

The integrals of (75) are

$$
\int \sqrt{4-(m+i n)^{2}} d t \pm \int \sqrt{4-(m-i n)^{2}} d t_{0}=\text { const.; }
$$

but these equations are equivalent to those obtained by equating to constants the real and imaginary parts of the function

$$
\int \sqrt{4-(m+i n)^{2}} d t
$$

If we substitute for $m+i n$ and $d t$ their values, we obtain the following result.

The directrix curves on both sheets of the focal surface correspond to the same orthogonal isothermal system of curves of the $\xi \eta$-plane or on the sphere, namely to the system obtained by equating to constants the real and imaginary parts of the function

$$
i \int \sqrt{4 w^{\prime}-\left[1+w^{\prime}+\frac{z-w}{2} \frac{w^{\prime \prime}}{w^{\prime}}\right]^{2}} \frac{d z}{z-w} .
$$

From this it follows, in particular, that these directrix curves are real. The same thing follows from the differential equation of the directrix curves when written in the form

$$
m n d \alpha^{2}-a d \alpha d \beta-m n d \beta^{2}=0,
$$

obtained from (75) by introducing the real variables $\alpha$ and $\beta$ in place of $t$ and $t_{0}$. For, the coefficients and variables in (77) are real, and the discriminant

$$
a^{2}+4 m^{2} n^{2}=\left(4-m^{2}+n^{2}\right)^{2}+4 m^{2} n^{2}
$$

is positive.

The osculating planes of the curves $\alpha=$ const. and $\beta=$ const. which meet at a point $P_{f}$ of $S_{f}$ intersect in a line through $P_{f}$ which is called the axis of $P_{f}$. The axes of all points of $S_{f}$ form a congruence called axis congruence, and the curves on $S_{f}$, which correspond to the developables of this congruence, are called its axis curves. The differential equation of the axis curves on $S_{f}$ are

$$
\left(m n-\frac{\partial^{2} \log m}{\partial \alpha \partial \beta}\right) d \alpha^{2}-\left(a-\frac{m_{a a}}{m}\right) d \alpha d \beta-m n d \beta^{2}=0 . \dagger
$$

* Sullivan, l.c., p. 194.

$\dagger \mathrm{E}$. J. Wilczynski, The general theory of congruences, these $\mathrm{Trans}$ a $\mathrm{cti}$ i $\mathrm{ns}$, vol. 16 (1915), p. 316, equation (9). 
The axis curves on $S_{g}$ are given by

$$
-m n d \alpha^{2}+\left(a+\frac{n_{\beta \beta}}{n}\right) d \alpha d \beta+\left(m n-\frac{\partial^{2} \log n}{\partial \alpha \partial \beta}\right) d \beta^{2}=0 .
$$

The Laplace transformation associates with every point of $S_{f}$ two points in the plane tangent to $S_{f}$ at $P_{f}$, namely the first and minus first Laplace transforms of $P_{f}$. The line joining these points is called the ray of $P_{f}$, and the totality of the rays of all points of $S_{f}$ is called its ray congruence. The ray curves of $S_{f}$, that is, the curves of $S_{f}$ which correspond to the developables of the ray congruence are given by

$$
-m n d \alpha^{2}+\left(a-\frac{m_{a a}}{m}\right) d \alpha d \beta+\left(m n-\frac{\partial^{2} \log m}{\partial \alpha \partial \beta}\right) d \beta^{2}=0 *^{*}
$$

Similarly we find as differential equation of the ray curves on $S_{\theta}$, the following:

$$
\left(m n-\frac{\partial^{2} \log n}{\partial \alpha \partial \beta}\right) d \alpha^{2}-\left(a+\frac{n_{\beta \beta}}{n}\right) d \alpha d \beta-m n d \beta^{2}=0 .
$$

The asymptotic curves on $S_{f}$ or $S_{g}$ are given by

$$
d t d t_{0}=d \alpha^{2}+d \beta^{2}=0 .
$$

Consequently, a relation of the form

$$
d \alpha_{1} d \alpha_{2}+d \beta_{1} d \beta_{2}=0
$$

between two tangents of $S_{f}$ at $P_{f}$ expresses the property of conjugacy. Clearly this relation exists between the surface tangents given by (79) and (81), and also between (80) and (82).

Therefore our congruence has the following additional property. On each sheet of the focal surface, each of its axis curve tangents is conjugate to one of the ray-curve tangents.

In order to study these relations a little more thoroughly we propose to find the equations of the directrices, axes, and rays of the points $P_{f}$ and $P_{g}$, referred to a common coördinate system. The most convenient system of coördinates for this purpose is one whose fundamental tetrahedron is made up of the four points $P_{f}, P_{g}, P_{\rho}$, and $P_{\sigma}$, where $P_{\rho}$ and $P_{\sigma}$ are defined by

$$
\rho=f_{a}-\frac{m_{a}}{m} f, \quad \sigma=g_{\beta}-\frac{n_{\beta}}{n} g
$$

$P_{\rho}$ and $P_{\sigma}$ are the first and minus first Laplace transforms of $P_{f}$ and $P_{g}$ with respect to the conjugate systems determined on $S_{f}$ and $S_{g}$ respectively by the developables of the congruence.

\footnotetext{
${ }^{*}$ L.c., p. 318 , equation (23).
} 
By direct application of the formulæ of the general theory of congruences we find the following results.*

The equations of the axis of $P_{f}$ are

$$
x_{3}=0, \quad x_{2}+\left(\frac{m_{\beta}}{m}-\frac{n_{\beta}}{n}\right) x_{4}=0 ;
$$

the axis of $P_{g}$ is given by

$$
x_{4}=0, \quad x_{1}-\left(\frac{m_{a}}{m}-\frac{n_{a}}{n}\right) x_{3}=0 .
$$

The ray of $P_{f}$ has the equations

and the ray $P_{g}$ is given by

$$
\begin{aligned}
& x_{1}=x_{4}=0, \\
& x_{2}=x_{3}=0 .
\end{aligned}
$$

To find the equations of the directrices of $P_{f}$ and $P_{g}$ referred to this coordinate system is not quite so easy. In the projective theory of surfaces, the equations of the directrices of $P_{f}$ are referred to a coördinate system whose vertices are given by $\dagger$

$$
\begin{gathered}
f, \quad \rho_{f}=f_{t}-\frac{1}{2} \frac{m_{t}}{m} f, \quad \sigma_{f}=f_{t_{0}}-\frac{1}{2} \frac{m_{t_{0}}}{m} f, \\
\tau_{f}=f_{t t_{0}}-\frac{1}{2} \frac{m_{t_{0}}}{m} f_{t}-\frac{1}{2} \frac{m_{t}}{m} f_{t_{0}}+\left[\frac{1}{4} \frac{m_{t} m_{t_{0}}}{m^{2}}-\frac{1}{4}\left(\frac{m_{t}}{m}\right)_{t_{0}}-\frac{1}{4}\left(\frac{m_{t_{0}}}{m}\right)_{t}\right] f,
\end{gathered}
$$

and it becomes necessary to obtain the equations of transformation between the two systems, which may be called the local coördinate systems of the surface $S_{f}$ and of the congruence. We find the following relations

$$
\begin{aligned}
& \rho_{f}=\frac{1}{4 m}\left(m_{a}+i m_{\beta}\right) f-\frac{i}{2} m g+\frac{1}{2} \rho, \\
& \sigma_{f}=\frac{1}{4 m}\left(m_{a}-i m_{\beta}\right) f+\frac{i}{2} m g+\frac{1}{2} \rho, \\
& \tau_{f}=\left[1+\frac{1}{4}\left(n^{2}-m^{2}\right)-\frac{1}{16} \frac{m_{a}^{2}}{m^{2}}+\frac{3}{16} \frac{m_{\beta}^{2}}{m^{2}}\right] f+\frac{1}{4}\left(2 m \frac{n_{\beta}}{n}-m_{\beta}\right) g \\
&-\frac{1}{4} \frac{m_{a}}{m} \rho+\frac{1}{2} m \sigma .
\end{aligned}
$$

Let $x_{1}, x_{2}, x_{3}, x_{4}$ be the coördinates of a point with respect to the system $f, g, \rho, \sigma$, so that the expression

\footnotetext{
${ }^{*}$ L. c., equations (5).

$\uparrow$ First Memoir, p. 248, equations (40).
}

$$
x_{1} f+x_{2} g+x_{3} \rho+x_{4} \sigma
$$


will furnish the homogeneous coördinates of the point referred to our fundamental homogeneous cartesian system when $f, g, \rho, \sigma$ are replaced by $f_{t}$, $g_{t}, \rho_{t}, \sigma_{t},(t=1,2,3,4)$. Similarly, let $x_{1}^{\prime}, x_{2}^{\prime}, x_{3}^{\prime}, x_{4}^{\prime}$ be the homogeneous coördinates of the same point referred to the local coördinate system of the surface $S_{f}$. Then the equation

$$
\omega^{\prime}\left(x_{1} f+x_{2} g+x_{3} \rho+x_{4} \sigma\right)=x_{1}^{\prime} f+x_{2}^{\prime} \rho_{f}+x_{3}^{\prime} \sigma_{f}+x_{4}^{\prime} \tau_{f},
$$

must be valid for all values of $f, g, \rho, \sigma$ after $\rho_{f}, \sigma_{f}, \tau_{f}$ have been replaced by their values (90); in this equation $\omega^{\prime}$ is an arbitrary factor of proportionality whose value is of no particular importance, on account of the homogeneity of our coördinate systems, and which may be given a special value so as to simplify the formulæ.

We obtain in this way the following equations of transformation:

$$
\begin{aligned}
\omega^{\prime} x_{1}=x_{1}^{\prime}+\frac{1}{4} \frac{m_{a}+i m_{\beta}}{m} x_{2}^{\prime} & +\frac{1}{4} \frac{m_{a}-i m_{\beta}}{m} x_{3}^{\prime} \\
& +\left(1+\frac{1}{4} n^{2}-\frac{1}{4} m^{2}-\frac{1}{16} \frac{m_{a}^{2}}{m^{2}}+\frac{3}{16} \frac{m_{\beta}^{2}}{m^{2}}\right) x_{4}^{\prime},
\end{aligned}
$$

(91) $\omega^{\prime} x_{2}=-\frac{i m}{2} x_{2}^{\prime}+\frac{i m}{2} x_{3}^{\prime}+\frac{1}{4 n}\left(2 m n_{\beta}-n m_{\beta}\right) x_{*}^{\prime}$,

$$
\begin{aligned}
\omega^{\prime} x_{3} & =\frac{1}{2} x_{2}^{\prime}+\frac{1}{2} x_{3}^{\prime}-\frac{1}{4} \frac{m_{a}}{m} x_{4}^{\prime}, \\
\omega^{\prime} x_{4} & =\frac{1}{2} m x_{4}^{\prime} .
\end{aligned}
$$

In computing the inverse of this transformation it is convenient to put $\omega^{\prime}=2 m$. We find

$$
\begin{aligned}
& x_{1}^{\prime}=2 m x_{1}+\frac{m_{\beta}}{m} x_{2}-m_{a} x_{3}+\left(m^{2}-n^{2}-4-\frac{1}{4} \frac{m_{a}^{2}+m_{\beta}^{2}}{m^{2}}-\frac{m_{\beta} n_{\beta}}{m n}\right) x_{4}, \\
& x_{2}^{\prime}=2 i x_{2}+2 m x_{3}+\left(\frac{m_{a}+i m_{\beta}}{m}-2 i \frac{n_{\beta}}{n}\right) x_{4}, \\
& x_{3}^{\prime}=-2 i x_{2}+2 m x_{3}+\left(\frac{m_{a}-i m_{\beta}}{m}+2 i \frac{n_{\beta}}{n}\right) x_{4}, \\
& x_{4}^{\prime}=4 x_{4} .
\end{aligned}
$$

Now, the equations of the directrix of the first kind of $P_{f}$ are

$$
x_{4}^{\prime}=0, \quad x_{1}^{\prime}+\frac{1}{2} \frac{A_{t}^{\prime}}{A^{\prime}} x_{2}^{\prime}+\frac{1}{2} \frac{B_{t_{0}}}{B} x_{3}^{\prime}=0,
$$


if we use the notations (73), and those for the directrix of the second kind are

$$
x_{2}^{\prime}+\frac{1}{2} \frac{B_{t_{0}}}{B} x_{4}^{\prime}=0, \quad x_{3}^{\prime}+\frac{1}{2} \frac{A_{t}^{\prime}}{A^{\prime}} x_{4}^{\prime}=0 . *
$$

Making use of (92) we find

$$
x_{4}=0, \quad x_{1}-\frac{m_{a}}{m} x_{3}=0
$$

as equations of the directrix of the first kind of $S_{f}$, and

$$
x_{3}=0, \quad x_{2}-\frac{n_{\beta}}{n} x_{4}=0
$$

for the directrix of the second kind of $S_{f}$, both referred to the local coördinate system of the congruence.

In order to obtain the corresponding equations for the directrices of $P_{g}$, we must supply some formulæ for the surface $S_{\sigma}$ which are analogous to certain corresponding formulæ for $S_{f}$, but which we have not noted so far. The equations of $S_{g}$ referred to the curves $\alpha=$ const. and $\beta=$ const. are

$$
\begin{aligned}
g_{a a}-g_{\beta \beta} & =2 \frac{n_{a}}{n} g_{a}+a g, \\
g_{a \beta} & =m n g+\frac{n_{\beta}}{n} g .
\end{aligned}
$$

The equations of $S_{\rho}$ referred to its asymptotic curves are

$$
\begin{array}{r}
g_{t t}-\frac{n_{t}}{n} g_{t}-\frac{n_{t}}{n} g_{t_{0}}+\frac{1}{4}\left[(m+i n)^{2}-4\right] g=0, \\
g_{t_{0} t_{0}}-\frac{n_{t_{0}}}{n} g_{t}-\frac{n_{t_{0}}}{n} g_{t_{0}}+\frac{1}{4}\left[(m-i n)^{2}-4\right] g=0 .
\end{array}
$$

The fundamental local coördinate system of $S_{o}$ is given by the four expressions

$$
\begin{gathered}
g, \quad \rho_{g}=g_{t}-\frac{1}{2} \frac{n_{t}}{n} g, \quad \sigma_{g}=g_{t_{0}}-\frac{1}{2} \frac{n_{t_{0}}}{n} g, \\
\tau_{g}=g_{t t_{0}}-\frac{1}{2} \frac{n_{t_{0}}}{n} g_{t}-\frac{1}{2} \frac{n_{t}}{n} g_{t_{0}}+\frac{3}{4} \frac{n_{t} n_{t_{0}}}{n^{2}} g
\end{gathered}
$$

so that

$$
\begin{aligned}
& \rho_{g}=\frac{1}{2} n f-\frac{n_{\alpha}+i n_{\beta}}{4 n} g-\frac{i}{2} \sigma, \\
& \sigma_{g}=\frac{1}{2} n f-\frac{n_{\alpha}-i n_{\beta}}{4 n} g+\frac{i}{2} \sigma,
\end{aligned}
$$

* Second Memoir, p. 95, equations (70a) and (70b). 
(100)

$$
\begin{aligned}
\tau_{g}=\frac{n}{4}\left(2 \frac{m_{a}}{m}-\frac{n_{a}}{n}\right) f-\left(1+\frac{1}{4} n^{2}-\frac{1}{4} m^{2}-\frac{3}{16} \frac{n_{a}^{2}}{n^{2}}\right. & \left.+\frac{1}{16} \frac{n_{\beta}^{2}}{n^{2}}\right) g \\
& +\frac{1}{2} n \rho-\frac{1}{4} \frac{n_{\beta}}{n} \sigma .
\end{aligned}
$$

If the coördinates of a point, $x_{1} f+x_{2} g+x_{3} \rho+x_{4} \sigma$, referred to this system are $x_{1}^{\prime \prime}, x_{2}^{\prime \prime}, x_{3}^{\prime \prime}, x_{4}^{\prime \prime}$, we find

$$
\begin{aligned}
& \omega^{\prime \prime} x_{1}=\frac{1}{2} n x_{2}^{\prime \prime}+\frac{1}{2} n x_{3}^{\prime \prime}+\frac{n}{4}\left(2 \frac{m_{a}}{m}-\frac{n_{a}}{n}\right) x_{4}^{\prime \prime}, \\
& \omega^{\prime \prime} x_{2}=x_{1}^{\prime \prime}-\frac{n_{a}+i n_{\beta}}{n} x_{2}^{\prime \prime}-\frac{n_{a}-i n_{\beta}}{n} x_{3}^{\prime \prime} \\
& \quad-\left(1+\frac{1}{4} n^{2}-\frac{1}{4} m^{2}-\frac{3}{16} \frac{n_{a}^{2}}{n^{2}}+\frac{1}{16} \frac{n_{\beta}^{2}}{n^{2}}\right) x_{4}^{\prime \prime}, \\
& \omega^{\prime \prime} x_{3}=\frac{1}{2} n x_{4}^{\prime \prime}, \\
& \omega^{\prime \prime} x_{4}=\frac{-i}{2} x_{2}^{\prime \prime}+\frac{i}{2} x_{3}^{\prime \prime}-\frac{1}{4} \frac{n_{\beta}}{n} x_{4}^{\prime \prime},
\end{aligned}
$$

whence$$
x_{1}^{\prime \prime}=\frac{n_{a}}{n} x_{1}+2 n x_{2}+\left(4+n^{2}-m^{2}-\frac{n_{a}^{2}+n_{\beta}^{2}}{4 n^{2}}-\frac{m_{a} n_{a}}{m n}\right) x_{3}-n_{\beta} x_{4},
$$$$
x_{2}^{\prime \prime}=2 x_{1}+\left(\frac{n_{a}+i n_{\beta}}{n}-2 \frac{m_{a}}{m}\right) x_{3}+2 n i x_{4},
$$$$
x_{3}^{\prime \prime}=2 x_{1}+\left(\frac{n_{a}-i n_{\beta}}{n}-2 \frac{m_{a}}{m}\right) x_{3}-2 n i x_{4},
$$$$
x_{4}^{\prime \prime}=4 x_{3} \text {. }
$$

From these formulæ we find

$$
x_{3}=0, \quad x_{2}-\frac{n_{\beta}}{n} x_{4}=0
$$

as the equations of the directrix of the first kind of $P_{g}$, and

$$
x_{4}=0, \quad x_{1}-\frac{m_{a}}{m} x_{3}=0
$$

for the directrix of the second kind of $P_{g}$. Comparison with (95) and (96) furnishes the following theorem:

The two focal sheets of our congruence are so related that the directrix of the first kind belonging to any point of either sheet is at the same time the directrix of the second kind of the corresponding point of the other sheet.

The University of Chicago, A pril 19, 1919. 